using a 'Merton nut' to remove its inevitable periodic errors. In the third stage, the corrected helix is covered with a thin layer of plastic which, when hardened and annealed, is removed from the cylinder and opened out upon an optically flat surface of swollen gelatine. When the whole has dried, the plastic 'pellicle' can be removed, and the underlying gelatine is found to be moulded into a flat replica of the corrected helix and can be hardened and aluminized.

It was shown by means of specimens and interferograms that these methods have already produced satisfactory gratings, particularly for work in the infra-red, and there seems every reason to hope that they will in due course yield large ghost-free gratings suitable also for emission spectroscopy. As it has been found impossible to devise a method of producing concave gratings from cylindrical matrices, a machine has been developed at the National Physical Laboratory for applying to a reciprocating concave blank a tool advanced by a 'Merton nut' driven by a screw of the pitch of the required grating.

Dr. A. C. Menzies, after referring to some recent work by Messrs. Hilger-Watts on the construction of fine-mesh metallic grids, went on to describe a modification of Sir Thomas Merton's process with the object of producing long gratings. This employs three successive operations. In the first, a short length of fine-pitch thread is engraved on a metal cylinder $B$ by a diamond driven from a 'Merton nut' riding on a similar cylinder $A$ on which a coarse thread has been engraved by a lathe. The two cylinders are connected together by a gear of ratio $32: 1$. In the second stage, a 'Merton nut' on $B$, with a diamond just ahead of it, extends the fine pitch thread as far as desired along the cylinder. The passage of the nut over the thread which it has ruled tends to produce irregular burnishing of the rulings. Although this would probably have little effect on the performance of a replica taken from the cylinder, it may be eliminated by ruling a third cylinder $C$ by means of a 'Merton nut' riding on $B$, the two cylinders being coupled together by a $1: 1$ gear. This cannot introduce periodic error, although it may give rise to slight non-linearity of the rulings, which, however, is regularly repeated and therefore not objectionable. In all cases, one end-bearing consists of a "Merton nut' on a 'neutral' thread, and the other of a similar nut on polished bar. A steel bar ruled by this method for a length of about nine inches was exhibited.

Dr. Menzies also directed attention to the probable value of the 'superfinishing' process, as used by Chryslers in the United States, in much reducing the time required to polish the metal cylinders preparatory to ruling by the Merton method.

In the discussion which followed, F. Twyman emphasized the desirability of comparative testing of existing gratings, especially some of the older ones reputed to be of 'good' quality. Prof. Curtis asked whether any correlation has yet been attempted between the results of the tests described by Mr. Guild and the performance of the grating in an actual spectrograph, to which Mr. Guild replied that this has not yet been possible as sufficient gratings of various qualities have not yet been available. Mr. Guild said that it is his object to establish such a correlation, and added that, although the tests might be capable of disclosing defects which are too slight to affect spectroscopic performance, such additional sensitivity is of great advantage in monitoring the development of the ruling process.
G. Sissen asked Mr. Guild whether it is possible by his wave-front shearing method to alter the inclination of the interfering wave-fronts, and consequently the spacing of the fringes. The latter replied that variation of fringe spacing is effected by displacing the double-image prism along the axis of the instrument. The direction of the fringes is, however, fixed relative to the direction of shear, in which respect Mr. Guild's beam divider is less flexible than that of Bates for general optical testing. Mr. Sissen then asked Dr. Sayce whether the thickness of the gelatine layer in the third stage of his process could be sufficiently increased to produce infra-red gratings, and received the reply that, although an increase in the thickness of the layer causes a slight departure from flatness, this is not enough to be detrimental in the infra-red region.

There was some discussion of the possibilities of the step-by-step method of grating ruling, which is attractive by reason of the elimination of the screw and its attendant errors. Various speakers referred to attempts on these lines, made in Germany, Sweden and Great Britain ; but it appears that none has been successful. The fundamental difficulty is thought to be that in any process of this type an accidental error of spacing will necessarily affect the whole of the subsequent rulings.

\section{THE 1951 EXHIBITION OF SCIENCE}

$T$

HE 1951 Exhibition of Science is splendidly housed, to the south of the Imperial College, in an extension of the Science Museum which is to form the nucleus of new permanent additions to the Museum buildings. A wealth of skill and decorative genius has gone to the making of a display which, despite some minor shortcomings, is an outstandingly good presentation of some of the main developments of pure science during recent years, with some appropriate historical background. It is planned as a continuous narrative (one might almost say a short course) and the round trip takes between one and two hours.

One enters by a maze-like tunnel with soundproofed bays, in each of which a loud-speaker suggests that one is shrinking to atomic dimensions as illuminated models of graphite crystals expand successively; at the end of the tunnel the visitor is on equal terms with an electron cloud. He is to see modern science from the atomic point of view.

The first stage of the journey around the exhibition itself deals with atomic structure. The diagrams and mural showing the history of the Periodic Table and its modern representation are good. The experiments on the electron are indifferent, chiefly due to lack of explanation as to why electric and magnetic deflexions occur ; and some of those listed are missing. There are some excellent animated diagrams illustrating radioactivity and nuclear physics, and two cloud chambers which work beautifully; but why the catalogue should include under radioactivity a spectrometer "capable of refractive index measurements of $0 \cdot 000001$ ", whatever that may mean, is a mystery.

Next come sections on chemical structure, the nature of chemical bonds, and the structure of metals and crystals. These are on the whole mag- 
nificent, lending themselves admirably to representation by the modeller. The exhibits on enzymes are introduced curiously-first by a film-strip of a rugby footballer which is quite irrelevant unless it is intended as a miming for the word 'conversion' (though it is a penalty kick), and then by a model of a dancing class which could not be interpreted; but the exhibits themselves are clear enough.

Associated with the chemical section are a number of poorly adjusted ripple-tank experiments, which do not do justice to a well-known make of apparatus, and which are intended to lead up to the idea of $\mathrm{X}$-ray diffraction by crystals. In this section also is shown the EMM electron microscope. The catalogue says this uses four magnetic lenses; as exhibited, it uses one electric lamp underneath one lantern slide, but nevertheless admonishes the public not to tamper with the controls. Is not a leading make of British electron microscope something to show proudly, in working order, on such an occasion ?

In the biological exhibits the emphasis is rather on living matter than on the organism as a whole; here again the modeller has had full scope and has done magnificently.

In the "Stop Press" section, pride of place must go to the exhibit on cosmic rays. This is fully documented, and tells the whole story from the first unaccountable electroscope leak to the most recent photographic plate work. It was worth the visit to see this exhibit alone, and to watch a counter-controlled chamber in action. Less impressive, but none the less excellent, is the smaller section on electrons in crystals, which explains the behaviour of luminescent materials clearly and effectively. Nimrod, the Ferranti electronic computer, is a good demonstration; the game it plays is simple, and any opponent who knows the rules has a good chance of success. The operator says it has a temper; but its face lights up after every move, whether advantageous or otherwise.

Nimrod, in fact, sets a standard by which many of the other 'working' exhibits might be judged. It really is in full working order, and it has someone in charge to explain what it is doing. Further, there is an excellent pamphlet published by Messrs. Ferranti, Ltd.*, which describes how it is constructed and how it works, and gives a little of the mathematical theory of digital computers. This pamphlet did not, however, seem to be available within the exhibition itself, which is a pity. There is a bookstall, at which expensive-looking commemorative jig-saw puzzles and scientific books quite unrelated to the exhibition are displayed; how much it could have contributed to the purpose of the whole if an explanatory pamphlet setting out details of some of the more striking individual exhibits (and moderately priced) had been on sale there! For the official guide-catalogue, useful as it is in explaining the exhibition as a whole, does not say very much that is helpful about individual items and is even confusing in places; in view of the fact that many items are neither clearly numbered nor effectively provided with displayed descriptive matter, this is a little disheartening. It says a great deal for the real quality of what there is on show that this criticism, which must be made, does not diminish one's admiration for the whole.

The writer was one of a small company of appreciative visitors who seemed to be pretty well out-

* Faster than Thought: the Ferranti Nimrod Digital Computer. Pp. 40. (Hollinwood: Ferranti, Itd., 1951.) 18. $6 d$. numbered by the attendants. By all accounts, the exhibition has not so far touched the popular imagination, and there may be several reasons for this. It is the academic conversazione on the grand scale, with the exhibits clearly linked by one central idea, the structure of matter. Perhaps this is the reason, for people will pay to be entertained or impressed, but not to be instructed. Its very success in achieving its aims may well limit its popular appeal. Or it may be that an enterprise of this kind takes a long time to become widely known and appreciated, or even found. It is quite possible to emerge from the South Kensington subway, and be inside the exhibition on the history of the Science Museum itself, before realizing that it is not what you were seeking; though, of course, there is some discreet short-range guidance for the reasonably observant.

One feels that there is something of permanent educational value here, and a pleasing creation which would be worth a visit if only to see a scientific exhibition dressed in a Festival gaiety.

G. R. NOAKES

\section{GERMAN SOCIETY FOR ELECTRON MICROSCOPY}

\section{THIRD ANNUAL CONFERENCE}

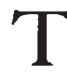

HE German Society for Electron Microscopy held its third annual meeting in Hamburg during May 18-20. Although the meoting was convened as a national conference, invitations had been accepted by many foreign men of seience, so that the conference took on a certain international flavour. Its success was due not only to the high quality of many of the contributions by German and foreign scientific workers, but just as much to the opportunities it offered to make, or renew, contacts between men and women of many countries.

The work of the conference was opened by $\mathrm{H}$. Ruska with a general lecture on the more recent results of electron microscopy, mainly illustrated by examples taken from the biological and medical field. Stress was laid on combining results obtained by electron microscopy with those obtained by other means.

Only some of the large number of papers read (about seventy) can be mentioned here. Mathematical investigations into the properties of certain magnetic electron lenses were presented by Lenz and by Glaser, and experimental methods for the field determination in magnetic lenses were described by Lenz and by Wolff. A very simple 'electron-optical' bench arrangement, to facilitate experimentation, was shown by Boersch.

Instrument design has been pursued steadily during the last year, and Möllenstedt described a simple way of increasing the specimen penetration in the electrostatic instrument by placing the specimen into a chamber kept at $+50 \mathrm{kV}$. with respect to earth, the gun operating at $-50 \mathrm{kV}$.; a direct switch-over from 50 to $100 \mathrm{kV}$. can be made. The use of the electron microscope in electron diffraction studies has been extended through the development by Heise of an adaptor for glancing incidence work with the AEG-Zeiss instrument. The design of a 